\title{
LIGHT-ACTUATED AC ELECTROOSMOSIS FOR OPTICAL MANIPULATION OF NANOSCALE PARTICLES
}

\author{
Pei-Yu Chiou, Aaron T. Ohta, Arash Jamshidi, Hsan-Yin Hsu, Jeffery B. Chou, and Ming C. Wu \\ Department of Electrical Engineering and Computer Sciences, University of California at Berkeley, \\ Berkeley, California, USA
}

\begin{abstract}
We report on a novel light actuated ac electroosmosis (LACE) mechanism enabling optical manipulation of nanoscale particles on a featureless photoconductive surface using direct optical images. This mechanism applied optically patterned microfluidic vortices to circulate the nanoscopic particles onto the surface of light patterned virtual electrodes and trap them via the strong electrokinetic forces near the surface. For particles larger than 1 $\mu \mathrm{m}$, we are able to achieve 31,000 individually addressable particle traps across a $1.3 \times 1 \mathrm{~mm}^{2}$ area. Each trap is created by one micromirror pixel of the digital-micromirror-devices (DMD) spatial light modulator used for patterning the optical images. For nanoscale particles, we have demonstrated optically guided concentration and transportation of $200 \mathrm{~nm}$ fluorescent nanoparticles.
\end{abstract}

\section{INTRODUCTION}

Tools for manipulating cells and particles in micro- and nanoscale play important roles in the biological and colloid fields. Manipulation functions such as trapping, concentrating, sorting, and transporting are highly sought after. Several mechanisms have been employed. For example, optical tweezers can precisely trap and transport a cell or a particle in a three-dimensional space[1]. Multiple traps can be generated using holography optical tweezers [2]. However, the optical power requirement and the small field of view of the high-N.A. objective lens limit its capability for parallel manipulation. Another commonly used mechanism is dielectrophoresis (DEP), which is produced by a non-uniform electric field[3]. Using microfabrication techniques, a large number of electrodes can be integrated for parallel manipulation. However, the size of the trap is limited by the electrodes, and its resolution is lower than that of optical tweezers. Parallel manipulation of a large number of single cells is even more challenging. It requires integrated "active matrix" circuits to individually address the electrodes, which increases the fabrication complexity and cost[4]. Magnetic tweezers and acoustic traps have also been reported $[5,6]$, however, it is difficult to trap and independently manipulate a large number of single cells.

To overcome this limitation, Chiou et al. have proposed a new mechanism called "optoelectronic tweezers (OET)," which allows optically patterned virtual electrodes on a photoconductive surface to address dielectrophoretic forces for massively parallel single cell manipulation using direct optical images[7]. By integrating a digital-micromirror-device (DMD) spatial light modulator, this technology allows a million light patterned virtual electrodes to be individually addressed on a low cost, disposable, plain amorphous silicon-coated glass for parallel control of a large number of single cells.

The DEP force is proportional to the volume and dielectric constant of the manipulated particles. Thus, a mixture of different particles can be sorted by DEP according to the characteristics of each particle because each particle may respond differently to the same electric field. However, manipulating nanoscale particles would require a very strong electric field gradient to overcome the thermal force. Alternatively, one can use fluidic flow to trap nanoparticles since the viscous force is only proportional to the linear dimension of the particles. As a result, manipulating particles using fluidic flow is much less dependent on the characteristics of the individual particles. Such a technique may be useful when it is desirable to manipulate each member of a mixture of particles in a substantially similar way.

In this paper, we present a novel mechanism called lightactuated ac electroosmosis (LACE) for manipulating micro- and nanoscale particles using light patterned microfluidic vortices. Unlike DEP, LACE uses fluidic flow to manipulate particles. Individually addressable traps for $2-\mu \mathrm{m}$ particles and concentration of $200-\mathrm{nm}$ particles have been demonstrated.

\section{THEORY}

Electroosmosis has been widely employed in the microfluidic systems for pumping liquids. The tangential electric field in the fluidic channel interacts with the charged ions in the double layer and generates a body force to drive the boundary fluidic layer. The velocity of the fluids at the edge of the double layer, called slip velocity, can be calculated by Helmholtz-Smoluchowski equation[8]

$$
v_{\text {slip }}=-\frac{\varepsilon \varsigma E_{t}}{\eta}
$$

where $v_{\text {slip }}$ is the slip velocity, $\varepsilon$ is the dielectric constant of the liquid, $\zeta$ is the zeta potential at the interface between the liquid and the channel, $E_{t}$ is the tangential component of the electric field, and $\eta$ is the fluidic viscosity. Electroosmosis has generally been shown in the dc electric field. Recently, the ac electroosmosis has also been demonstrated as an effective mechanism in pumping fluidic flow by coupling the tangential electric field component with the field induced double layer charges on the electrode[9]. (a)

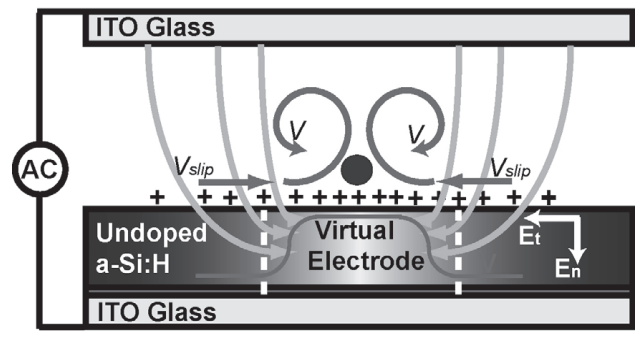

(b)

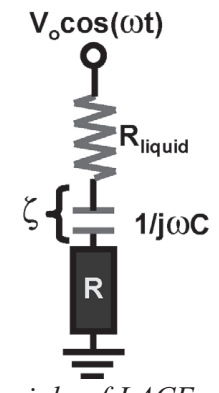

Fig.1 (a) Schematic illustrating the operating principle of LACE.. (b) The equivalent circuit model along one of the electric field lines.

Figure 1(a) illustrates the device structure and the working principle of LACE. The liquid solution containing nanoparticles are sandwiched between an indium tin oxide (ITO) glass and a photoconductive surface consisting of multiple featureless layers 
including an ITO glass, a 50-nm n+ a-Si:H layer, and an 1- $\mu \mathrm{m}-$ thick undoped a-Si:H layer. An ac bias is applied between the top and the bottom ITO glass. In the dark area, the electric impedance of the amorphous silicon layer is high, and only a small portion of the total voltage drops across the liquid layer. In the illuminated area, the increased photoconductivity creates a virtual electrode on amorphous silicon and causes the applied bias to drop across the interface double layer capacitor $\mathrm{C}$ and the liquid resistor, as shown in Fig. 1(b). By using light patterning to create virtual electrodes, electrode patterns can be changed quickly and efficiently, rendering fixed physical or chemical pattering of the electrodes unnecessary.

Since creating the boundary slip velocity requires both the tangential electric field and the charged ions at the interface, there exists an optimal ac frequency $\mathrm{f}_{\mathrm{opt}}=1 / 2 \pi \mathrm{RC}$ at which the product of the electric field and the interface zeta potential in Eq. (1) reaches maximum. For frequency much high than $\mathrm{f}_{\text {opt }}$, the capacitor has low electrical impedance and the bulk liquid resistor dominates. This results in a small zeta potential and a small slip velocity. For frequency much lower than $f_{\text {opt }}$, the impedance of the capacitor is much larger than the bulk liquid layer. The electric field that can penetrate through the double layer is small, resulting in a small tangential electric field. This also yields a small slip velocity. It can be shown that the optimal frequency of the device structure shown in Fig.1 (a) is

$$
f_{\text {opt }}=\frac{1}{2 \pi} \frac{\sigma}{\varepsilon} \frac{\lambda_{d}}{L}
$$

where $\sigma$ is the conductivity of the liquid medium, $\varepsilon$ is the dielectric constant of the liquid, $\lambda_{d}$ is the double layer thickness, and $L$ is the gap spacing of the liquid layer. For a LACE device with a $100 \mu \mathrm{m}$ gap spacing, a KCL electrolyte with a liquid conductivity of 10 $\mathrm{mS} / \mathrm{m}$ and a double layer thickness around $10 \mathrm{~nm}$, the estimated $\mathrm{f}_{\text {opt }}$ is equal to $229 \mathrm{~Hz}$. The actually $\mathrm{f}_{\text {opt }}$ is slightly higher when considering geometry factor due to the diverging electric field pattern in the liquid layer. In current LACE device, the ac frequencies are usually between $1 \mathrm{kHz} \sim 10 \mathrm{kHz}$, while DEP operates at much higher frequencies, above $20 \mathrm{kHz}$ to $10 \mathrm{MHz}$, to decouple the ac electroosmosis effect. The liquid conductivity is between $3 \mathrm{mS} / \mathrm{m} \sim 10 \mathrm{mS} / \mathrm{m}$.

The microfluidic vortex created by the light induced ac electroosmosis near the virtual electrode is illustrated in Fig.1 (a). The charged ions accumulated at the interface are attracted towards the center by the tangential field, carrying the surrounding fluids with them. This results in a slip velocity pointing towards the center of the virtual electrode. By projecting a symmetrical optical pattern, a balanced microfluidic vortex is generated at the virtual electrode.

Trapping of nanoscopic particles using LACE involves two processes. First, the circulating fluid brings the nanoscopic particles via viscous forces to the center of the virtual electrode, where a minimum speed of the flow pattern exists since there is no tangential electric field due to symmetry. Second, although there is no tangential electric field in the center of the virtual electrode, the normal component of the electric field is strong. This results in the trapping of nanoparticles on the surface by the strong electrokinetic forces in the vertical direction, either through particle induced electrohydrodynamic flow or DEP forces.

\section{SIMULATION}

The LACE mechanism is simulated by combining the electrostatic model with the incompressible Navior Stoke's fluidic model. A de electrostatic model in FEMLAB 3.0 is used to calculate the zeta potential, the tangential component of the electric field, and the slip velocity at the surface of the photoconductive layer. To facilitate computation, the double layer capacitor is replaced by a conductive layer with the same magnitude of frequency dependent resistance. Fig. 2 shows the vertical and the tangential components of the electric field distribution in the liquid layer using the following parameters, double layer thickness: $10 \mathrm{~nm}$, liquid conductivity: $10 \mathrm{mS} / \mathrm{m}$, applied bias: $2 \mathrm{~V}$. The equivalent resistance of the capacitor is calculated at $8 \mathrm{kHz}$. The photoconductivity in the a-Si:H layer is a gaussian distribution with a full-width-at-half-maximum (FWHW) size of $30 \mu \mathrm{m}$ and a peak conductivity $0.2 \mathrm{mS} / \mathrm{m}$. Fig. 2(a) shows the vertical electric field is symmetric with respect to the center of the illumination spot and has a strong gradient near the surface. This provides the strong electrokinetic forces to trap nanoparticles at the surface. The tangential electric field on the surface is antisymmetric, as shown in Fig. 2(b). The zeta potential can be extracted out from the voltage across the capacitor layer and the slip velocity at the interface is calculated using Eq. (1) along the radial direction. This slip velocity is used as the boundary condition to solve the incompressible Navior Stoke's equations for the flow pattern shown in Fig. 3.
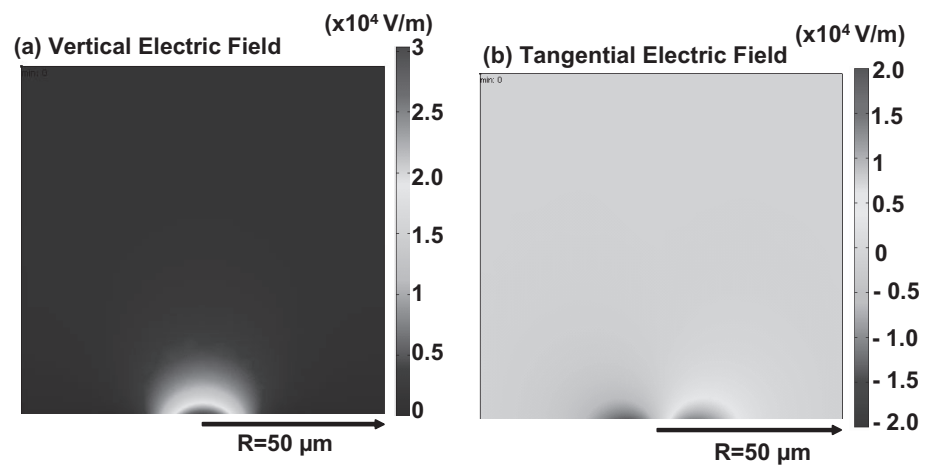

Fig.2 (a) Vertical and (b) tangential electric field distributions in the liquid layer.

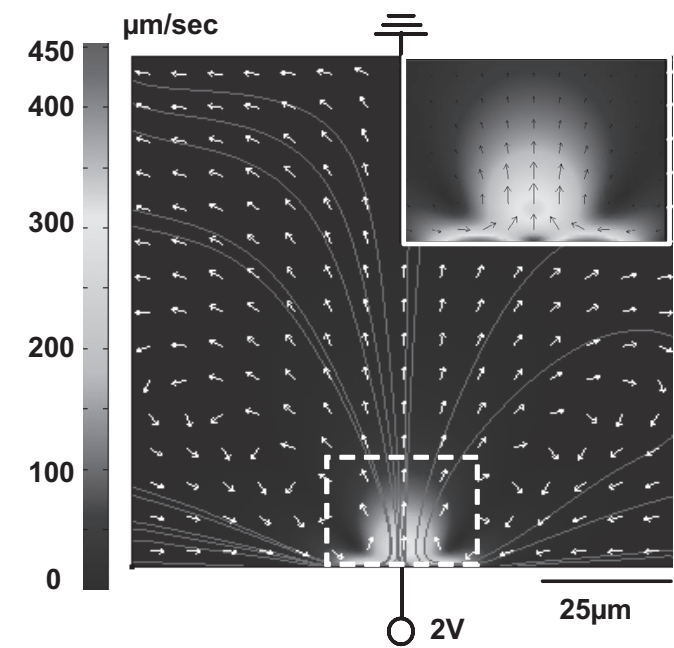

Fig.3 Light induced fluidic flow near the virtual electrode. The inset shows the enlarged flow pattern in the center. There exists a dead zone in the middle where nanoparticles are trapped.

In Fig. 3, this light induced flow circulates around the illumination spot with the highest speed at the edge of the virtual electrode. In the center surface of the virtual electrode, there exists a dead zone where the flow speed reaches minimum, as shown in 
the inset in Fig. 3. The nanoparticles are circulated and swept into the dead zone where the electrokinetic forces dominate over the fluidic viscous forces and trap the nanoparticles at the center surface of the virtual electrode.

\section{DEVICE FABRICATION AND EXPERIMENTAL SETUP}

Figure 4 shows the pictures of the fabricated device. Fig. 4(a) is the photoconductive glass with multiple featureless layers as described in Fig. 1(a). The a-Si:H near one edge is removed by reactive ion etching (RIE) $\left(\mathrm{CF}_{4}+\mathrm{O}_{2}\right)$ to expose the bottom ITO layer for electrical contact. Fig. 4(b) is the commercial available transparent ITO glass.

(a)

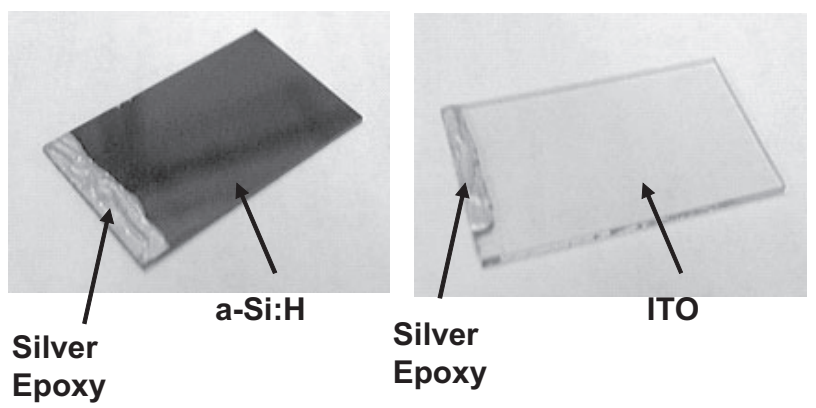

Fig.4 Pictures of the LACE device. (a) The a-Si:H-coated ITO glass. The a-Si:H layers at the edge is removed for electrical contact. (b) The commercial available ITO glass.

Figure 5 shows the experimental setup constructed on a Nikon TE-2000E inverted microscope. The LACE device is placed on the observation stage. We can operate the LACE device with the photoconductive side either up or down. For fluorescent observation, the photoconductive surface is placed on top of the ITO glass to prevent absorption of the excitation and the emission signals by amorphous silicon. This configuration is used for nanoparticle experiments since a fluorescent signal is used to observe particles smaller than the optical diffraction limit.

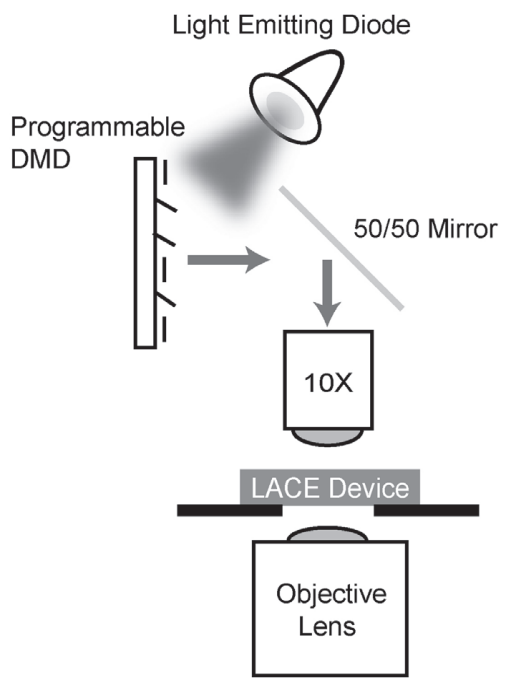

Fig.5 The experimental setup for nanoparticle manipulation using LACE.
The optical patterns are projected from the top side to minimize the interference with observation microscope. In this paper, we used two kinds of optical sources. The first is a single light-emitting diode (LED) with a wavelength of $625 \mathrm{~nm}$ (Lumileds, Luxeon Star/O). The light is expanded and spatially modulated by a DMD with $769 \times 1024$ mirror pixels. Each pixel has a size of $13.68 \times 13.68 \mu \mathrm{m}^{2}$. The optical image is projected onto the LACE device using a $10 \times$ objective lens, generating a $1.5 \times 1.5$ $\mu \mathrm{m}^{2}$ optical pixel on the imaging plane and an optical power of 25 $\mathrm{nW} /$ pixel. In some experiments, the background fluorescent excitation intensity is too strong, the LED does not provide sufficient contrast. In that case, a $5-\mathrm{mW}, 632-\mathrm{nm}$ laser beam is directly focused on the LACE surface by the $10 \times$ objective lens.

\section{EXPERIMENTAL RESULTS}

We have created 31,000 optical traps across a $1.3 \times 1 \mathrm{~mm}^{2}$ area for trapping and transporting $2-\mu \mathrm{m}$ beads in parallel. Fig. 6 shows a small part of the trap array. Each optical trap is produced by a single DMD pixel. In Fig. 6(a), the particles are randomly distributed and not trapped by LACE yet. Within a second, these particles are swept into the bright spots by the microfluid vortices induced around the virtual electrodes. The spot size of the virtual electrode is small enough that each trap can only accommodate one particle. The un-trapped particles keep moving in the dark area until they are captured by an empty trap, as shown in Fig. 6(b). The applied ac frequency is $1 \mathrm{kHz}$ and the bias is $4 \mathrm{~V}_{\mathrm{pp}}$. The process from Fig. 6(a) to (c) takes less than 10 seconds. The same optical patterns can also be used to trap $1 \mu \mathrm{m}$ beads individually. For particles with size smaller than $1 \mu \mathrm{m}$, multiple particles will be trapped in one optical spot.
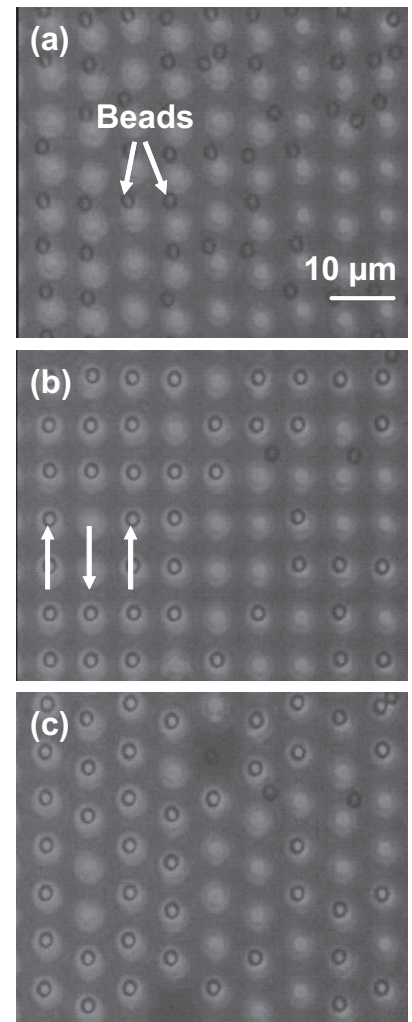

Fig. 6 Parallel trapping and transport of single $2 \mu$ m polystyrene beads. Each trap is produced a single DMD mirror pixel and the pitch is 5 pixels. 

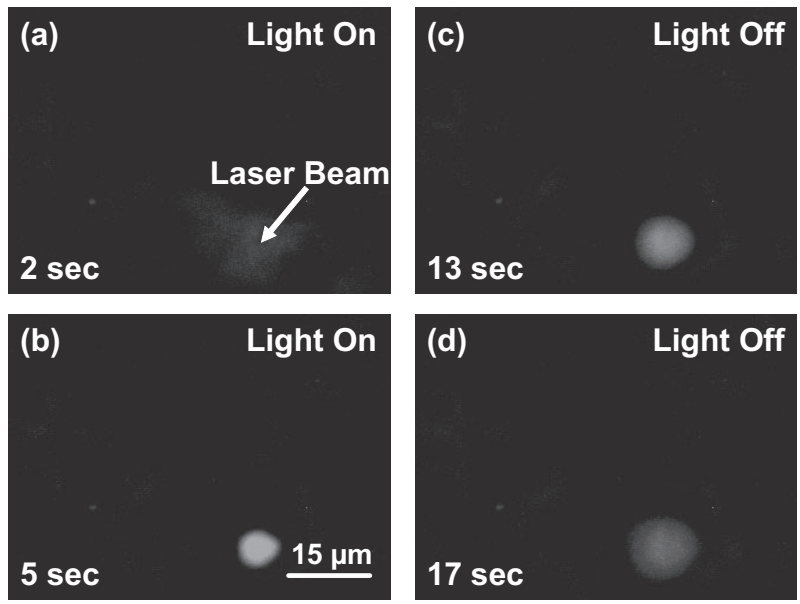

Fig.7 The snapshots of the captured video showing the concentration and release of 200- $\mathrm{nm}$ fluorescent particles using LACE activated by a 5-mW, $632 \mathrm{~nm}$ laser beam focused by a $10 \times$ objective lens.

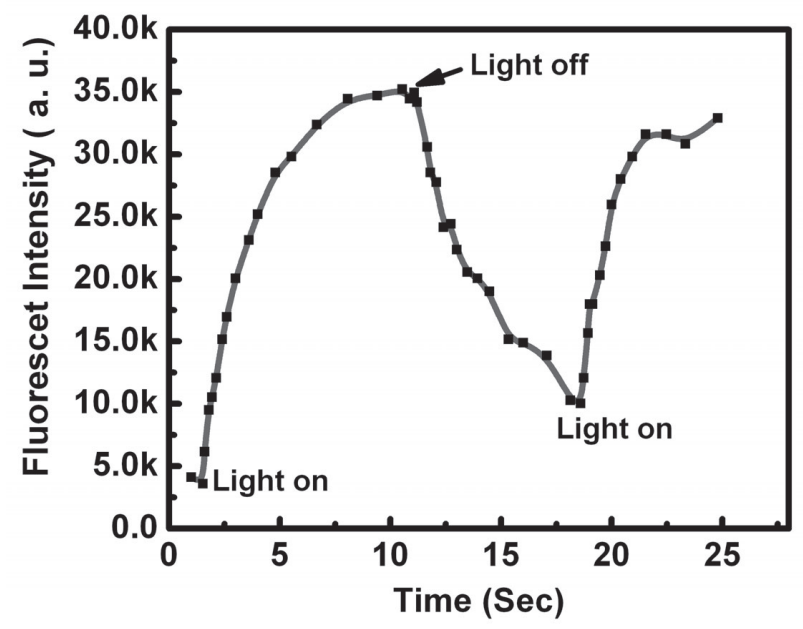

Fig.8 The fluorescent intensity at the center of the illumination spot. The concentration and releasing process of nanoscopic particles is repeatable.

Figure 7 shows the trapping and releasing of a group of 200 $\mathrm{nm}$ green fluorescent particles using a $5 \mathrm{~mW}, 632 \mathrm{~nm}$ laser beam and a $8.3 \mathrm{~V}_{\mathrm{pp}}, 1.6 \mathrm{kHz}$ ac bias. In Fig. 7(a), the particles are dispersed in the solution and the fluorescent signals are very weak. In Fig. 7(b), we turn on the focused laser beam near the lower right corner. The particles are concentrated to the center of the illuminated spot and produce a strong fluorescent signal. The fluorescent intensity saturated after few seconds, indicating the number of trapped particles has reached the maximum. The laser beam is turned off after the intensity saturated. These concentrated particles start to diffuse away due to the Brownian motion and the intensity of the fluorescent signal goes back to the background intensity after a few seconds, as shown in Fig. 7(c) and (d). This concentration and releasing process is repeatable. Fig. 8 shows the temporal response of the fluorescent signal at the center of the illumination spot.

\section{CONCLUSIONS}

This paper presents a novel light-induced ac electroosmosis (LACE) mechanism that enables optically patterned microfluidic flow to manipulate particles ranging from $2 \mu \mathrm{m}$ to $200 \mathrm{~nm}$ on a featureless photoconductive glass. In contrast to DEP, which uses electric field to exert forces directly on particles according to their dielectric constant, LACE uses electric field to cause fluidic flow, which can then be used to trap particles, essentially independent of their dielectric constants. Using a DMD spatial light modulator, the high-resolution virtual electrodes generated 31,000 individually addressable microfluidic vortices to trap and transport $2 \mu \mathrm{m}$ and $1 \mu \mathrm{m}$ particles. For particles smaller than $1 \mu \mathrm{m}$, we have achieved light activated nanoparticle concentration using the LACE mechanism. This light-activated concentration and releasing process is controllable and repeatable. These experimental results show that LACE is potentially a powerful technique to manipulate nanoscale particles optically in real time.

\section{ACKNOWLEDGEMENT}

This project is supported by Center for Cell Mimetic Space Exploration (CMISE), a NASA University Research, Engineering and Technology Institute (URETI). P.Y. Chiou is partially supported by UC GREAT Fellowship.

\section{REFERENCES}

[1] A. Ashkin, J. M. Dziedzic, J. E. Bjorkholm, and S. Chu, "Observation Of A Single-Beam Gradient Force Optical Trap For Dielectric Particles," Optics Letters, vol. 11, pp. 288-290, 1986.

[2] J. E. Curtis, B. A. Koss, and D. G. Grier, "Dynamic holographic optical tweezers," Optics Communications, vol. 207, pp. 169-175, 2002.

[3] M. P. Hughes, "Strategies for dielectrophoretic separation in laboratory-on-a-chip systems," Electrophoresis, vol. 23, pp. 2569-2582, 2002.

[4] N. Manaresi, A. Romani, G. Medoro, L. Altomare, A. Leonardi, M. Tartagni, and R. Guerrieri, "A CMOS chip for individual cell manipulation and detection," IEEE Journal Of Solid-State Circuits, vol. 38, pp. 2297-2305, 2003.

[5] H. Lee, A. M. Purdon, and R. M. Westervelt, "Manipulation of biological cells using a microelectromagnet matrix," Applied Physics Letters, vol. 85, pp. 1063-1065, 2004.

[6] H. M. Hertz, "Standing-Wave Acoustic Trap For Nonintrusive Positioning Of Microparticles," Journal Of Applied Physics, vol. 78, pp. 4845-4849, 1995.

[7] P. Y. Chiou, A. T. Ohta, and M. C. Wu, "Massively parallel manipulation of single cells and microparticles using optical images," Nature, vol. 436, pp. 370-372, 2005.

[8] J. Lyklema, Fundamentals of Interface and Colloid Science, vol. 2. London: Academic Press, 1991.

[9] A. Ramos, H. Morgan, N. G. Green, A. Gonzalez, and A. Castellanos, "Pumping of liquids with traveling-wave electroosmosis," Journal Of Applied Physics, vol. 97, 2005. 